\title{
Bioanalysis: 10 years of progress
}

\author{
Neil Spooner*,1 \\ ${ }^{1}$ Spooner Bioanalytical Solutions Ltd, Hertford, SG138BQ, UK \\ *Author for correspondence: neil@spoonerbioanalytical.co.uk
}

\section{"Over the 10 years since its founding, Bioanalysis has become the go to 'house' journal for the bioanalytical community."}

First draft submitted: 7 March 2019; Accepted for publication: 12 March 2019; Published online: 4 April 2019

Keywords: anniversary • bioanalysis • community

Welcome to this special anniversary issue of Bioanalysis, celebrating the first 10 years of this most impactful publication for the bioanalytical community. Bioanalysis - the Journal first appeared online in late March 2009, with the print versions becoming available in April 2009 [1]. This first issue ran to a bumper 22 articles over 253 pages. The content of this issue comprised Editorials from the Senior Editors Brian Booth and Howard Hill, News \& Analysis articles, a White Paper, a challenge article, six research articles and nine reviews. These manuscripts covered many themes that are still vibrant and familiar to the bioanalytical community now in 2019, including microsampling, microanalysis, 'omics, alternative separations (HILIC, chiral, UPLC), stability and cross company groups reaching consensus. It is notable that articles on approaches for the analysis of small molecules by LC-MS dominated the issue.

Since that time, the discipline that we term 'bioanalysis' has changed in many ways. While small molecule LC-MS still dominates in 2019, other areas have grown and evolved. Novel structural modalities, such as peptides, biopharmaceuticals, oligonucleotides and novel constructs such as antibody-drug conjugates have become progressively more prominent in the portfolios of pharmaceutical companies. In addition, the determination of changes in the concentrations and distributions of endogenous molecules has become increasingly important to enable us to understand the efficacy and safety of therapeutic agents ('omics, biomarkers, antidrug antibodies, etc.). There has also been a development in the options that are available for the collection of high-quality blood and plasma samples for the quantitative analysis of drugs, metabolites and biomarkers (DBS, microsampling), leading to ethical and data quality benefits and the ability to collect previously intractable samples, often in locations that are more convenient to the subject than conventional phlebotomy. These different analytes and sample types have in turn led to changes in the analytical tools that are required by the bioanalytical laboratory, for example, immunoassays, digestion LC-MS, immuno-precipitation, qPCR, flow cytometry, high-resolution MS, rapid chromatography, capillary separations, MALDI and so on. In turn, this change has required different skill sets for bioanalytical scientists, often requiring increased biological skills in addition to proficiency in traditional analytical chemistry. This requires the retraining of existing staff, or the recruitment of new with a different skill base. These different approaches have also required alternative considerations for the validation and application of the analytical methods and the measurement of analytes. In many cases, the rules developed for the analysis of small-molecule drugs are not useable for these new ways of working, resulting in active discussions in the pages of this journal. Another major change that has come to fruition during the first 10 years of publishing the Journal Bioanalysis, is the increase in outsourcing of bioanalytical work by pharmaceutical companies to CROs. In many cases, pharma companies now have little, or no in-house bioanalytical capability and are increasingly relying on the expertise of their CRO partners. The journal has kept in constant step with these changes, reflecting and supporting the science and the increasingly diverse bioanalytical community.

It is significant that from its inception, the journal not only took the line of publishing high-quality research and perspectives, it also took steps to broaden the appeal of the articles and to encourage the authors to consider the wider and future implications of their work. This included highlighting particular significant references, summarizing the work in an extended executive summary and giving a future perspective for their work. All of these are still notable 
and welcome features of the journal 10 years later, a testament to the foresight of the founding Editorial team and Senior Editors (Brian Booth and Howard Hill). The journal soon proved to be successful, with its combination of cutting-edge research and community building White Papers and Conference Proceedings. From an initial one issue per month at launch in April 2009, the journal soon expanded to two issues a month in January 2011. This adds-up to a mighty 213 issues of the journal that have been published up to the end of 2018 .

From the outset, the journal has been differentiated from many other publications in the field, in that it has a full-time Editorial Staff who are able to keep the content current and the agenda flowing. Just as unique and important are the broader Editorial Advisory Board, who bring a wealth of real-world bioanalytical experience to the publication and enrich it with their wisdom and generosity.

A key feature of Bioanalysis has been the publication of themed issues, focusing on a particular topic that is of current and ongoing interest to the community. From 2009 to the end of 2018, there have been 63 special issues, approximating to a third of the journals published output in this time. Topics have included new tools and techniques (e.g., DBS, green bioanalysis, electronic laboratory, sample preparation, sample separation, highresolution MS, imaging and accelerator MS), developing application areas (e.g., 'omics, biomarkers, biotherapeutics, novel drug constructs, tissue analysis, automation and metabolism) and working practices and regulations (ISR, tiered approach, matrix effects and outsourcing). These focused special focus issues have brought together stateof-the-art science and opinion from the leading and upcoming investigators in the particular area of interest. Furthermore, they provide readers with an up-to-date insight into the many areas that make up the field of bioanalysis and enable future perspectives on their development to be shared.

Another important contribution to the content of the journal has come from Conference Reports, White Papers and Consensus Documents, particularly from sources such as WRIB, EBF, CPSA, Reid Forum, GCC, CBF and JBF. In fact, these articles tend to be among those most read in the journal. These manuscripts are often written by multiple authors from different organizations and give an up-to-date, concise and consensus insight into how the field of bioanalysis is developing, particularly for technology, applications and regulatory aspects. They also reflect the increasingly global and diverse nature of bioanalytical science.

In 2011, the publishers of Bioanalysis launched a companion website - Bioanalysis Zone [2]. Through this unique pairing, the community has gained an avenue for creating and consuming content in a format that is not amenable to traditional printed media. This has included among others, panel discussions, webinars, expert opinions, interviews, surveys, infographics and educational content. It has also enabled the recognition of the leading lights in the field through awards such as the Bioanalysis Rising Star Award and Bioanalysis Outstanding Contribution Award. Importantly, much of the Bioanalysis Zone content has been accompanied by articles in the journal, where more in-depth analysis and supporting material can be provided. Together, these two media provide a high-quality, accessible, inclusive and up-to-date platform for the field.

Over the 10 years since its founding, Bioanalysis has become the go to 'house' journal for the bioanalytical community. Its pages have contained a narrative to the changes and developments that have occurred in the science and regulations, which are central to its readers. It is important that the journal maintains its high quality, and scientific and editorial integrity to enable it to build upon its successes into the future. The permanent editorial staff, editorial board and most importantly the readership will be pivotal in helping to keep the journal on course and relevant to our community.

This tenth anniversary special issue of bioanalysis brings all of the above content together with contributors from a wide spectrum of professions for whom bioanalysis is the basis of their day-to-day work and how they identify themselves professionally. These include those from pharma, biotechs, CROs, academia, central labs, government labs, vendors, regulators and many others. I want to give thanks to the authors who have taken their precious time to consider the past and future impact of our 'house' journal and the broad-based field of bioanalytical science that it represents. Their collective passion and insight are pivotal to our joint journey and success of this community. Bioanalysis Journal enables us to work together and build this community for the benefit of patients and those for who we generate important decision making data, most of who have never even heard of bioanalysis!

As we begin the second decade of the journal, I find it interesting to speculate on what bioanalytical science and its associated community might look like in 2029. I think we will see the pace of change in healthcare quicken, with ever more emphasis on the needs of the patient/consumer. This will encourage bioanalytical scientists to think in a very different way to our current emphasis on technologies and workflows that best suit the bioanalytical scientist. A major benefit of this change will be that the bioanalytical community will more often look outside their own areas of expertise to learn how other communities are able to deliver a product and solutions to the required standards. 
Regardless of how this all plays out, I am certain that bioanalytical science will look very different to today and that the technologies, applications, the community and those that it serves will keep evolving and broadening in scope. New drug modalities, novel analytical approaches, automation, big data and changes in regulations will all be part of the story. Also, and most importantly, the consideration of the human patient/consumer as central to all that we do will grow in importance. I am certain that the journal will also continue to change and develop to reflect this. I personally cannot wait to be part of the journey and to see where it takes us. Watch this space!

\section{Disclaimer}

N Spooner is the Senior Editor of Bioanalysis.

\section{Financial \& competing interests disclosure}

The author has no relevant affiliations or financial involvement with any organization or entity with a financial interest in or financial conflict with the subject matter or materials discussed in the manuscript. This includes employment, consultancies, honoraria, stock ownership or options, expert testimony, grants or patents received or pending, or royalties.

No writing assistance was utilized in the production of this manuscript.

\section{References}

1 Launch issue. Bioanalysis www.future-science.com/toc/bio/1/1

2 Bioanalysis Zone. www.bioanalysis-zone.com 
